inflammatory processes at the cellular level, it is highly speculative on the part of the authors to extrapolate these data to infections. It is also important to remember that during an infection, what may be food for the host could also represent a more accessible source of nutrient for the pathogen. For example, it is known that the metabolism of $M$. tuberculosis in its host is geared to the processing of fatty acids as a key carbon source ${ }^{10}$. Although these latter speculations may lack experimental support, the observations reported in this study lead us to a greater appreciation of the role of lipids in driving phagosome maturation, and its subversion by infectious agents.

1. Russell, D. G. Nature Rev. Mol. Cell Biol. 2 , 569-577 (2001).

2. Anes, E. et al. Nature Cell Biology 5, 793-802 (2003).

3. Guerin, I. \& de Chastellier, C. Infect. Immun. 68 2655-2662 (2000)

4. Vieira, O. V., Botelho, R. J. \& Grinstein, S. Biochem J.
366, 689-704 (2002).

5. Fratti, R. A., Backer, J. M., Gruenberg, J., Corvera, S. \& Deretic, V. J. Cell Biol. 154, 631-644 (2001).

6. Fratti, R. A., Chua, J., Vergne, I. \& Deretic, V. Proc. Natl Acad. Sci. USA 100, 5437-5442 (2003).

7. Malik, Z. A. et al. J. Immunol. 170, 2811-2815 (2003).

8. Spiegel, S. \& Milstien, S. FEBS Lett. 476, 55-57 (2000).

9. Schaible, U. E., Sturgill-Koszycki, S., Schlesinger, P. H. \& Russell, D. G. J. Immunol. 160, 1290-1296 (1998).

10. McKinney, J. D. et al. Nature 406, 735-738 (2000).

\section{Tip of the POP2}

The process of pollination in flowering plants has been broadly understood for some time. Pollination begins when haploid male pollen grains are deposited in the diploid female pistil. After germination, the pollen grain forms a tube that extends through the style to reach the transmitting tract. The tube emerges from the tract and reaches the septum, where the ovules are attached, before locating the micropyle opening of the ovule. Tube growth is then arrested and fertilization of the egg cell occurs.

Although the basic biology of pollen tube growth is understood, the molecular mechanisms underpinning the process are relatively unclear. Now, a study by Palanivelu, R. et al. (Cell 114, 47-59 (2003)) sheds new light on the process by demonstrating that pollen tube navigation is controlled by a gradient of the amino acid $\gamma$-amino butyric acid (GABA). In higher plants, GABA has previously been linked with regulation of $\mathrm{pH}$, nitrogen storage, development and pathogen defence.

Palanivelu et al. now identify a connection with reproduction by characterizing the Arabidopsis thalania POP2 gene, mutation of which is known to cause late-stage defects in pollen tube transport through the pistil. Intriguingly, they find that $P O P 2$ encodes a transaminase that regulates steady-state levels of GABA, and to a lesser extent $\beta$ alanine. This prompted examination of GABA levels in wild-type and pop2 mutant plants. In wild-type plants, an increasing concentration of GABA along the direction of pollen tube growth was identified: that is, from the stigma, through the style to the ovule. Furthermore, there is a particularly high GABA concentration in the inner integument cells, which flank the micropyle. In pop2 mutants, however, there is an approximately 100 -fold general increase in GABA levels that masks the GABA gradient. In many instances, pollen tube growth was abolished, in other examples, growth towards ovules was mis-guided (see figure), possibly through attraction to ectopically expressed GABA found on the ovule surface. Furthermore, in an interesting twist, the

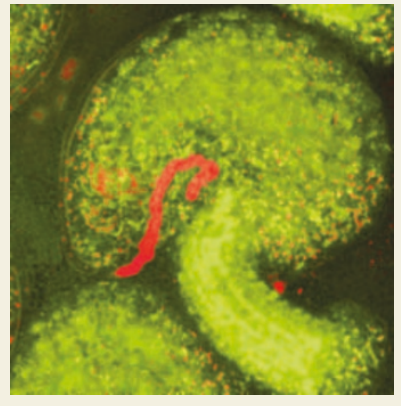

A mis-targeted pollen tube (red) from a pop2 mutant growing inappropriately on the ovule surface (green).

authors show that wild-type tubes grow normally through pop2 pistils, suggesting that excess GABA is perhaps degraded by the end of the pollen tube to reveal the underlying gradient.

Thus, the authors conclude that GABA stimulates pollen tube growth, that high concentrations inhibit pollen tube elongation and that it functions as a signal to guide pollen tube navigation, most probably in conjunction with other guidance cues. One obvious extension of these studies will be to identify the pathways that regulate GABA signalling. On this note, the authors point out that they could not identify Arabidopsis GABA receptor genes similar to those found in animals, although the abundance of GABA in plants suggests that plant-specific receptors must exist. In conclusion, however, Palanivelu et al. provide the first concrete evidence to link GABA signalling to pollen tube growth and infertility.

DONALD MCDONALD 